
\title{
25 Research Soure \\ Diagnostic and Therapeutic Errors in Classical Trigeminal Neuralgia: a Multicenter Survey
}

Fabio Antonaci ( $\square$ fabio.antonaci@mondino.it)

https://orcid.org/0000-0001-6206-1237

Sebastiano Arceri

IRCCS Mondino Foundation

Martin Rakusa

University Medical Centre, Marbor

Dimos D. Mitsikostas

National and Kapodistrian University of Athens

Ivan Milanov

St Naum Hospital

Vasil Todorov

St Naum Hospital

Matteo Cotta Ramusino

IRCCS Mondino Foundation

Alfredo Costa

IRCCS Mondino Foundation

\section{Research article}

Keywords: trigeminal neuralgia, therapeutic mistakes, diagnostic mistakes, clinical errors, guidelines

Posted Date: March 3rd, 2020

DOI: https://doi.org/10.21203/rs.3.rs-15716/v1

License: (c) (1) This work is licensed under a Creative Commons Attribution 4.0 International License.

Read Full License 


\section{Abstract}

Background Classical Trigeminal neuralgia (CTN) is a severe, disabling form of painful cranial neuropathy. Even though CTN has a typical clinical picture, diagnosis it is often missed or delayed in clinical practice. This study was carried over to investigate the occurrence of diagnostic and therapeutic errors in CNT.

Methods We studied 102 patients suffering from CTN recruited through a multicentric survey. All the patients were evaluated using telephone/frontal interviews conducted by headache/pain specialists using an ad hoc questionnaire.

Results Patients were mostly females (F:M ratio 2.64:1); in 50\% of cases age ranged between 51 and 70 years. Eighty-six percent of the patients consulted a physician at the time they experienced the first pain attacks. Specialists consulted before CTN diagnosis was finally made were: primary care physicians (43.1\%), dentists (in $30.4 \%$ of cases), otorhinolaryngologists (3.9\%), neurosurgeons (3.9\%), neurologists or headache specialists $(14.7 \%)$, others $(8 \%)$. In most cases $(85.3 \%)$ the final diagnosis was made by a neurologist or headache specialist, and the mean interval between the disease onset and the specialist consultation was $10.8 \pm 21.2$ months. The correct diagnosis were made after $7.2 \pm 12.5$ months, being the misdiagnoses at the first consultation in $42.1 \%$ of cases. Ten patients underwent erroneous dental extraction. Instrumental and laboratory investigations were carried out in $93.1 \%$ of the patients before the final diagnosis of CTN.

Conclusions Our results emphasize the need to improve the neurological knowledge in order to promptly recognize the clinical picture of CTN and properly adhere to the specific treatment guidelines.

\section{Background}

Classical Trigeminal neuralgia (CTN) is a severe, disabling form of painful cranial neuropathy. According to the beta version of the 3rd edition of the International Classification of Headache Disorders (ICHD-3 Beta), CTN is "characterized by recurrent unilateral brief electric shock-like pains, abrupt in onset and termination, limited to the distribution of one or more divisions of the trigeminal nerve and triggered by innocuous stimuli. It may develop without apparent cause or be a result of another diagnosed disorder"[1]. Trigeminal neuralgia is classified as idiopathic occurred without apparent cause; classical caused by vascular compression of the trigeminal nerve root, or secondary, mainly caused by demyelinating lesions (e.g. multiple sclerosis) or space occupying lesions [2]. The diagnosis of CTN requires the absence of a clinically evident neurological deficit, such as hypoaestesia or hypoalgesia occurring in trigeminal regions, that may be indicative of a trigeminal neuropathy. The clinical manifestations of CTN usually involve the second and third branch of the trigeminal nerve and the pain is always unilateral. The typical aspect of paroxysmal attacks is the refractory period in which the pain cannot be evoked. 
The intensity of the attacks produces a psychosocial dysfunction, significantly impairing quality of life and for this reason these patients require a prompt diagnosis followed by an appropriate treatment $[3,4]$. Furthermore, the suboptimal neuropathic pain management contributes to the significant association between pain severity and poorer health status [4]. However, even though CTN has a typical clinical picture, diagnosis is often missed or delayed in clinical practice. To the best of our knowledge there is no previous report in literature on diagnostic and therapeutic errors in CTN. Therefore, we designed a multicenter hospital-based study in order to investigate diagnostic and therapeutic errors in CTN sufferers, along the temporal pattern of the disease.

\section{Methods}

As in a previous study by our group on trigeminal autonomic cephalalgias [5], we performed a Pubmed database search using the following combination of terms: trigeminal neuralgia AND errors OR pitfalls OR misconception OR delay OR mismanagement OR misdiagnosis OR underdiagnosed. No peer-reviewed scientific contribution to the field was found at the time of publication.

Patients were then enrolled consecutively in the several Headache Centers involved in the study. Diagnosis of CTN was made according to the International Headache Society (IHS) criteria [1]. In the period from February 2017 to October 2019, 102 patients were recruited on first consultation or follow-up in the Centers participating in the study (most of patients in Italy, then Bulgaria, Greece, Croatia, Egypt, Serbia, Albania, Denmark, Romania). The patients were invited to take part in a face to face or telephone interview conducted by a qualified headache specialist, using an ad hoc questionnaire (Fig. 1). The questionnaire was developed by authors and was the final result of a dedicated consensus meeting of the members of the Pain Panel and Headache Panel of the European Academy of Neurology (EAN). It was a 18-item questionnaire designed to assess the demographic data, the type of specialist consulted, the time elapsed between the first attacks and the first diagnosis/correct diagnosis, the knowledge (if any) of the existence of dedicated headache centers, the investigations carried out, and any medications prescribed and/or taken. After that the questionnaire was administered, the patients interviewed became aware of their CTN diagnosis.

Statistics

The results were assembled in a database and analyses were carried out using SPSS (version 21.0; SPSS, Chicago, IL, USA).

\section{Results}

Demographic data

The study included 102 patients with CTN, 74 females and 28 males (F:M ratio 2.64:1). Of these, 1\% were less than 30 years old, $23.5 \%$ were aged between 31 and 50 years, $50 \%$ between 51 and 70 years, and 
25.5\% were more than 70 years old. Most patients were retired (33.3\%), the rest were employees (49\%), unemployed (10.7\%), and employers (7\%).

Consulted physicians

Most of patients ( $n=88,86.3 \%$ ) had consulted a physician at the time of the first pain attacks. The remaining patients $(n=14,13.7 \%)$ had not sought medical aid after the first episodes (so called "patient delay"). Figure 1 reports the distribution of the physicians consulted at the first visit: approximately half of patients had seen a primary care physician $(n=44,43.1 \%)$, and nearly one third a dentist $(n=31$, $30.4 \%)$. The other consulted specialists were: neurosurgeons $(n=4 ; 3.9 \%)$, otolaryngology (ENT) ( $n=4$; $3.9 \%)$, ophthalmologists $(n=1 ; 1 \%)$, rheumatologists $(n=1 ; 1 \%)$, emergency doctors $(n=1 ; 1 \%)$, physiotherapist $(n=1 ; 1 \%)$, while surprisingly only 15 patients $(14.7 \%, 2$ of which were headache specialists) had referred to a neurologist (Fig. 2). Only 18 patients (17.6\%) received a correct diagnosis at the first consultation. It is worth noting that 84 patients $(82.4 \%)$ consulted a second physician before a correct diagnosis was obtained, 38 patients (37.2\%) a third specialist, and 12 patients $(11.8 \%)$ even a fourth one. The specialists seen on a second consultation were: neurologists $(n=41 ; 48.7 \%)$ and headache specialists $(n=5 ; 6.0 \%)$, dentists $(n=18 ; 21.4 \%)$ ENT doctors $(n=12 ; 14.2 \%)$, primary care physicians $(n=3 ; 3.6 \%)$, neurosurgeons $(n=2,2.4 \%)$, ophthalmologists $(n=2 ; 2.4 \%)$, others $(n=1 ; 1.2 \%)$. The third consultation was made by the following: neurologists $(n=20 ; 52.6 \%)$, headache specialists $(n=$ $7 ; 18.4 \%)$, ENT doctors $(n=5 ; 13.2 \%)$, dentists $(n=2 ; 5.3 \%)$, ophthalmologists $(n=2 ; 5.3 \%)$, neurosurgeons $(n=1 ; 2.6 \%)$ and maxillo-facial surgeons $(n=1 ; 2.6 \%)$. When a fourth consultation had been necessary to reach a diagnosis, the consulted specialist were a neurologist $(n=11)$ and in only one case a general practitioner. One patient, in spite of having received the correct diagnosis of CTN by a neurologist at first consultation, had decided to ask a dentist for a second opinion.

Most of the patients consulted two physicians $(n=45 ; 44.1 \%)$ prior to obtain a correct diagnosis, while 26 patients (25.5\%) consulted three physicians, 12 patients (11.7\%) four physicians, and only 19 patients (18.7\%) consulted one physician (Fig. 3). Eighty patients (78.4\%) were already aware to suffer from CTN before submitting the questionnaire. Only few patients $(n=22,21.6 \%)$ were diagnosed with CTN at the time of submission of the questionnaire.

We found that generally CTN diagnosis had been made by a neurologist $(n=72 ; 70.6 \%)$, or a headache specialist $(n=15 ; 14.7 \%)$. In a few cases, diagnosis was received by dentists $(n=6 ; 5.9 \%)$, neurosurgeons $(n=6 ; 5.9 \%)$, and only in three cases by primary care physicians $(n=3 ; 2.9 \%)$. Unfortunately, $49 \%$ of the patients interviewed were not aware of the existence of Headache Centers, or other specific structures dedicated to the treatment of headache and cranial neuralgias. Furthermore, $18.6 \%$ of the interviewed patients claimed to have self-diagnosed CTN on the basis of different sources of information (Internet $n=$ 16 , scientific books $n=1$, or talk with other people suffering from CTN, $n=2$ ), before seeking medical confirmation. Thirty-one patients $(31.4 \%)$ in our study also suffered from another type of headache (migraine without aura $n=18$, tension type-headache $n=12$; cluster headache $n=1$; Horton' arteritis $n=$ 1). 
The mean interval between onset of the disease and specialist consultation ("Patient Delay") in a Headache Center was 10.8 \pm 21.2 months (range $0-144$ months). In Italy, delay was $8.02 \pm 14.2$ months, whereas in other European Centers was up to $12.6 \pm 25.05$ months. In our cohort, 2 outlier patients (Fig. 4) influenced significantly the average delay: one patient was indeed diagnosed after 72 months and another one after as long as 12 years. Three of the interviewed patients did not receive any headache specialist evaluation. The average time between disease onset and a correct diagnosis ("Diagnostic Delay") was $7.2 \pm 12.5$ months (in Italy $8.4 \pm 12.8$ months, in the other European centers $7.13 \pm 13.01$ months). Only in one patient no information was obtained. Misdiagnoses at first consultation were reported in 43 cases (42.1\%), while 40 subjects (39.2\%) did not receive a diagnosis during the visit; only 19 subjects (18.4\%) received a correct one. Only one patient, despite obtaining the correct diagnosis, asked for a second specialist consultation. In the group of patients $(n=84)$ who underwent a second consultation, 28 patients (33.3\%) were misdiagnosed, 11 patient $(13.1 \%)$ did not receive a definite diagnosis, while 45 patients (53.6\%) were not diagnosed correctly, although one received the indication for a nonspecific treatment with corticosteroids. In the group facing a third evaluation $(n=38), 6$ subjects $(15.8 \%)$ did not obtain a specific diagnosis, 6 patients received a misdiagnosis, while 26 patients $(68.4 \%)$ received a correct diagnosis (15.8\%) (Fig. 5).

The different diagnoses received before the correct one of CTN were also analyzed. The total number of misdiagnoses was 77 (mean number of diagnosis per patient: 0.75). Misdiagnoses on the first consultation were 43 , on the second 28 , and on the third 6 . Overall, the reported misdiagnoses and related frequency were the following: dental problems $(n=37 ; 48 \%)$, including toothache, periodontal abscess, dental caries, dental granulomas; sinusitis $(n=11 ; 14.3 \%)$; unspecific facial pain $(n=7 ; 9.1 \%)$; unspecified headache $(n=6 ; 7.8 \%)$; migraine $(n=5 ; 6.5 \%)$; cluster headache $(n=4 ; 5.2 \%)$; temporomandibular joint dysfunction $(n=3 ; 3.9 \%)$; and tension-type headache $(n=1 ; 1.3 \%)$, glaucoma ( $n$ $=1 ; 1.3 \%)$, otitis $(n=1 ; 1.3 \%)$ tonsillitis $(n=1 ; 1.3 \%)$.

Investigations

Instrumental and laboratory investigations prior to establish a CNT diagnosis had been carried out in almost all cases (95 patients; $93.1 \%$ ). Most of patients ( $n=70 ; 73.7 \%$ ) had undergone a brain MRI in order to detect a possible neurovascular conflict or other causes of symptomatic TN; 40 patients (42.1\%) had undergone a CT scan, 12 patients $(12.6 \%)$ a skull X-rays, 8 patients $(8.4 \%)$ an orthopantomography, 8 patients $(8.4 \%)$ a blink reflex test, 2 patients $(2.1 \%)$ a spine X-Ray, 2 patients $(2.1 \%)$ a carotid ultrasound imaging, one patient (1\%) an electroencephalogram (EEG). More than one instrumental examination had been performed in 37 patients (38.9\%). In 25 patients $(26.3 \%)$ some investigations (skull X-ray, orthopantomography, EEG, carotid ultrasound imaging, spine X-ray) appeared to be unnecessary [68].

\section{Treatment}

In our sample, 19 patients $(18.6 \%)$ had not received any symptomatic treatment before diagnosis. The remaining patients had been prescribed symptomatic drugs, especially analgesics, such as NSAIDs $(n=$ 
$69 ; 67.6 \%)$ and opiates $(n=19 ; 18.6 \%)$. In the latter group, 13 patients used opiates in association with NSAIDs. Then, 8 patients had been prescribed gabapentinoid drugs (gabapentin 100mg/day or pregabalin $150 \mathrm{mg} /$ day), 3 patients had been treated with triptans as needed. Other treatments were: antibiotics $(n=1)$, benzodiazepines $(n=1)$, B vitamin supplements $(n=2)$, mannitol i.v. $(n=2)$, verapamil $(n=1)$, duloxetine $(n=1)$, topiramate $(n=1)$. Seven patients claimed to be on treatment with unspecified anti-epileptic drugs.

After that the correct diagnosis was established, the first-choice treatment was: carbamazepine in $80.3 \%$ $(n=82)$, gabapentinoid drugs in $11.7 \%(n=12)$, topiramate in $2 \%(n=2)$, lamotrigine in $2 \%(n=2)$; oxacarbazepine in $1 \%(n=1)$, methylprednisolone in $1 \%(n=1)$, opiates in $1 \%(n=1)$, antidepressants not better specified in $1 \%(n=1)$. In different phases of the disease, some patients switched to another pharmacological regimen, with the addition/substitution of a second drug. In 10 patients $(9.8 \%)$ the drug introduced was lamotrigine, a choice due to partial/complete inefficacy of the first treatment, but the most frequent treatments chosen as second option were gabapentinoids $24.5 \%(n=25)$, in addition/substitution to the first one. The other associated treatments were: unspecified antidepressants 6.8\% $(n=7)$; carbamazepine 3.9\% $(n=4)$, oxacarbazepine 1.9\% $(n=2)$, venlafaxine $1.9 \%(n=2)$, duloxetine $(n=1)$. Five unresponsive patients had been treated with amitriptyline as a third choice. It is worth noting that 18 patients received a treatment not included in the dedicated guidelines for TN $[6,8]$, i.e. topiramate $(n=2)$, methylprednisolone iv $(n=1)$, opiates $(n=1)$, amitriptyline $(n=5)$, duloxetine $(n=$ $1)$, unspecified antidepressants $(n=8)$.

Before CTN diagnosis, $17.6 \%$ of the patients $(n=18)$ had already undergone some treatment procedures, such as tooth extraction ( $n=10 ; 55.5 \%$ ), in 2 cases even multiple, or treatments not recommended by guidelines, i.e acupuncture $(n=4 ; 22.2 \%)$; local injection of steroids $(n=2 ; 1.9 \%)$, anesthetics and botulinum toxin $(n=1)$, Thecar therapy $(n=1)$. Only 1 patient had undergone 3 subsequent microvascular decompression surgeries, and later a balloon compression and a percutaneous glycerol rhizolysis of Gasserian ganglion. Eighty-two patients (80.4\%) had followed the therapy prescribed after the diagnosis, whereas 20 subjects $(19.6 \%)$ had interrupted the treatment, due to the following reasons: side effects (in particular dizziness and asthenia) $(n=1)$, uneffectiveness $(n=17)$, leukopenia $(n=1)$, allergic reaction $(n=1)$.

\section{Discussion}

In the present study, we evaluated the occurrence of misdiagnosis and errors in the management of patients suffering from CTN. To our knowledge, this is the first investigation in the field, whereas some reports on the same issue are available in literature with regard to primary and secondary headaches.

The demographic data of our sample are consistent with previous studies reporting a greater prevalence of CTN in females (F: M ratio 2.64:1) [9]. The evaluation of the data obtained revealed that about half of the patients $(42.1 \%)$ had received a wrong diagnosis at the first medical evaluation, while only $14.2 \%$ had been correctly classified as suffering from CTN. Moreover, the mean interval between the onset of 
symptomatology and the actual diagnosis exceeded 7 months. This finding appears to be similar in all the centers involved in the study, suggesting a similar general approach to diagnostic criteria of TN.

Interestingly, we also observed that in order to obtain a correct diagnosis, patients had to contact a mean of two specialists. While the latency between the first access of patients to health resources and the correct diagnosis may appear significant, it has to be said that the mean diagnostic delay in patients with a primary headache like cluster headache $(\mathrm{CH})$ was reported to be even greater (more than 5 years) in a recent study [5]. This could be explained by different factors: CTN is probably more known in the medical field, and patients suffering from CTN show a sharp-triggered pain with daily pattern of several attacks [10].

During their full blown phase of disease, CTN patients in our study had consulted several doctors, but in most cases $(85.3 \%)$ the diagnosis had been made by a neurologist or a headache specialist. This confirms that while the general practitioner often represents the first medical figure that patients consult at the first presentation of pain attacks CTN remains a strictly specialistic diagnosis. Moreover, in our study we noted that due to the topographic distribution of the pain (II or III branch of the trigeminal nerve) and the characteristics of the pain itself (sharp, stabbing) the first specialist consulted had been the dentist, in the suspicion of a dental pathology [11]. This had resulted in several cases of misdiagnosed underlying pathology, and hence in unnecessary dentistry procedures, such as dental extractions. However, the delay in the diagnosis also by other specialists implies a mismanagement of the disease. Garvan et al showed that $73 \%$ of patients with TN, in an attempt to control the pain, received unnecessary dental assessment, and of these, more than $60 \%$ underwent dental extraction (for a total of 680 teeth extracted) [12]. These data are in line with those later obtained by other authors reporting in a retrospective study that more than $80 \%$ of CTN patients had referred to a dentist for new onset trigeminal symptoms, and that $66 \%$ of them had received unnecessary dental treatment, including extractions (mean of 2 teeth), root canal treatments and implants, before consulting a neurologist [13].

It is interesting to compare these data to those regarding $\mathrm{CH}$, a primary headache form that is frequently misdiagnosed as TN. Our results appear indeed to be in contradiction with a previous study on diagnostic and therapeutic errors in $\mathrm{CH}$ where the first specialist consulted was the neurologist (48.6\%), the dentist being seen only in $2.8 \%$ of the cases, and the misdiagnosis of dental problems had occurred in $4.2 \%$ of cases [5]. The different clinical presentation of $\mathrm{CH}$ in bouts compared to TN may explain such discrepancy.

In our study, more than half of the patients (62.7\%) had to consult two doctors before receiving the correct diagnosis. As suggested by EAN guidelines on TN, a MRI of the brain and brainstem is recommended to exclude a symptomatic form of disease [7]. In our study, $73.7 \%$ of patients had undergone a brain MRI study and some of them also a brain CT scan, along with other unnecessary examinations such as orthopanthography, EEG, spine X-rays, skull X-rays, and carotid ultrasound imaging [7]. 
It is worth underlining that before the diagnosis, patients had been formally prescribed or were taking symptomatic drugs according to a self -medication regimen, with little or no benefit, especially from the NSAID class and opioids for acute attacks. Only a minority of patients had been prescribed anti-epileptic drugs, of the class of gabapentinoids, before the diagnosis. In addition, 3 patients had been adviced to assume triptans for the acute attack, without benefit. Then, after the correct diagnosis, in agreement with the available guidelines, carbamazepine and oxcarbamazepine were introduced as a first-line treatment [7]. Other drugs used as a first-line therapy, either in single-dose or in combination, were gabapentinoids, lamotrigine and topiramate. These observations are in agreement with current evidence-based guidelines on TN treatment from the EAN which consider carbamazepine as the first-choice treatment for TN $[7,14]$. From the analysis of our data, it would therefore appear that the limiting factor in the workup and management of TN is the diagnostic delay.

According to the mentioned guidelines the indication for surgery is a condition in which pain is not sufficiently controlled medically or a medical treatment is poorly tolerated [7]. Patients should be informed of such possibility at an early stage. Although surgical treatment for TN is generally effective, the important complications of the different procedures limit its use as a first-line option: for example percutaneous procedures on Gasserian lesions can cause facial sensory loss (painful anesthesia). Gamma-knife appears to be the least invasive and the safest procedure, but pain relief may take long to develop [7]. In our patients, drugs not formally recommended for TN, such as amitriptyline, duloxetine, venlafaxine and in one case parenteral methylprednisolone, had been introduced as add-on therapy. However, once the diagnostic workup has been set up and treatments have been prescribed in accordance with to the international guidelines, therapeutic errors should not occur anymore $[6,7]$.

\section{Conclusion}

This study suggests that while CTN has typical features and it is well defined by the available international criteria, it is still frequently misdiagnosed and mistreated. To avoid this, a tight cooperation on the basis of a continuous medical education between neurologists, general practitioner, dentists and neurosurgeons appears to be mandatory when evaluating a patient with orofacial pain suggesting TN. Larger population studies may provide further evidence in order to identify the correct strategies to reduce delays due to both patients and professionals, and expand our knowledge on the overall management of this disease.

\section{Abbreviations}

$\mathrm{CH}=$ cluster headache; $\mathrm{CT}$ = computed tomography; $\mathrm{CTN}$ = classical trigeminal neuralgia; $\mathrm{EAN}=$ European Academy of Neurology; EEG = electroencephalogram; ENT = Otolarynologist (Ear, Nose, Throat); IHS = International Headache Society; NSAID = non-steroidal anti-inflammatory drug.

\section{Declarations}


Ethics approval and consent to participate

The study was approved by the local Ethics Committees and every patient provided a written informed consent.

Consent for publication

Not applicable

Availability of data and materials

The datasets used and/or analysed during the current study are available from the corresponding author on reasonable request.

Competing interests

The authors declare that they have no competing interests

Funding

This study was supported by a research grant from the Italian Ministry of Health (2012) to IRCCS Mondino Foundation, Pavia, Italy. Collaboration with the Italian Society for the Study of Headache (SISC), section of Lombardia, is also gratefully acknowledged.

Authors' contributions

Study conception and design is due to FA. FA, SA, MR, DDM, IM and VT performed the clinical consultations and participated in the data collection. FA and SA interpreted the data, and together with AC and MCR wrote the manuscript. All authors reviewed critically and approved the final manuscript.

Acknowledgements

We are grateful to G. Cruccu (Rome, Italy), and N. Attal (Boulogne-Billancourt, France) for their advice in protocol design, and to: G. Dalla Volta, D. Carli (Brescia, Italy), T. Maikova (Kiev, Ukraine), V. Kosta (Split, Croatia) A. Radojicic (Belgrade, Serbia), M. Z. Gomaa (Sohag, Egypt) and J. Kruja (Tirana, Albania) for their contribution in patients' recruitment.

\section{References}

1. Headache Classification Committee of the International Headache Society. The International Classification of Headache Disorders, 3rd edition (beta version). Cephalalgia 2013;33(9):629-808. doi: $10.1177 / 0333102413485658$

2. Cruccu G, Finnerup NB, Jensen TS, Scholz J, Sindou M, Svensson P, Treede RD, Zakrzewska JM, Nurmikko T. Trigeminal neuralgia: New classification and diagnostic grading for practice and 
research. Neurology 2016;87(2): 220-8. doi: 10.1212/WNL.0000000000002840

3. Allsop MJ, Twiddy M, Grant H, Czoski-Murray C, Mon-Williams M, Mushtaq F, Phillips N, Zakrzewska JM, Pavitt S. Diagnosis, medication, and surgical management for patients with trigeminal neuralgia: a qualitative study. Acta Neurochir. 2015;157 (11):1925-33. doi: 10.1007/s00701-015-2515-4

4. Tölle T, Dukes E, Sadosky A. Patient burden of trigeminal neuralgia: results from a cross-sectional survey of health state impairment and treatment patterns in six European countries. Pain Pract. 2006;6(3):153-60.

5. Voiticovschi-losob C, Allena M, De Cillis I, Nappi G, Sjaastad O, Antonaci F. Diagnostic and therapeutic errors in cluster headache: a hospital-based study. J Headache Pain.2014;15:56. doi: 10.1186/1129-2377-15-56

6. Attal N, Cruccu G, Baron R, Haanpää M, Hansson P, Jensen TS, Nurmikko T. EFNS guidelines on the pharmacological treatment of neuropathic pain: 2010 revision. Eur J Neurol. 2010;17:1113-23. doi: 10.1111/j.1468-1331.2010.02999.x

7. Bendtsen L, Zakrzewska JM, Abbott J, Braschinsky M, Di Stefano G, Donnet A, Eide PK, Leal PRL, Maarbjerg S, May A, Nurmikko T, Obermann M, Jensen TS, Cruccu G. European Academy of Neurology guideline on trigeminal neuralgia. Eur J Neurol. 2019;26(6): 831-49. doi: 10.1111/ene. 13950

8. Cruccu G, Gronseth G, Alksne J, Argoff C, Brainin M, Burchiel K, Nurmikko T, Zakrzewska JM. AANEFNS guidelines on trigeminal neuralgia management. Eur J Neurol. 2008;15:1013-28. doi: 10.1111/j.1468-1331.2008.02185.x

9. Maarbjerg S, Di Stefano G, Bendtsen L, Cruccu G. Trigeminal neuralgia - diagnosis and treatment. Cephalalgia. 2017;37(7):648-57. doi: 10.1177/0333102416687280

10. Viana M, Tassorelli C, Allena M, Nappi G, Sjaastad O, Antonaci F. Diagnostic and therapeutic errors in trigeminal autonomic cephalalgias and hemicrania continua: a systematic review. J Headache Pain. 2013;14:14. doi: 10.1186/1129-2377-14-14

11. Horowitz M, Ochs M, Carrau R, Kassam A. Trigeminal neuralgia and glossopharyngeal neuralgia two orofacial pain syndromes encountered by dentists. J Am Dent Assoc. 2004;135(10):1427-33.

12. Garvan NJ, Siegfried J. Trigeminal neuralgia- earlier referral for surgery. Postgrad Med J. 1983;59(693):435-7.

13. Von Eckardstein KL, Keil M, Rohde V. Unnecessary dental procedure as a consequence of trigeminal neuralgia. Neurosurg Rev. 2015;38(2):355-60. doi: 10.1007/s10143-014-0591-1

14. Obermann M. Treatment options in trigeminal neuralgia. Ther Adv Neurol Disord. 2010;3(2):107-15. doi: $10.1177 / 1756285609359317$

\section{Figures}


ean

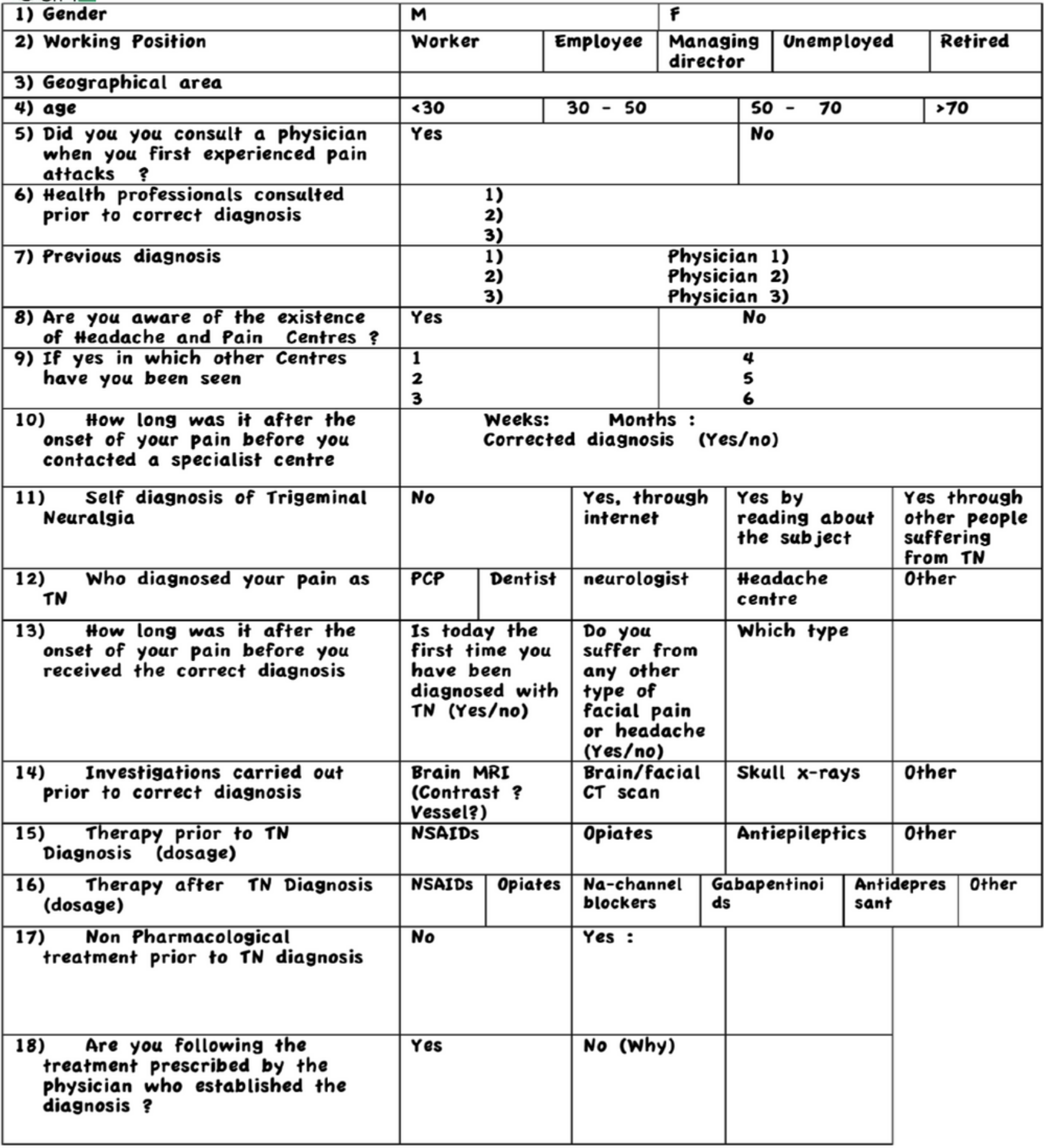

\section{Figure 1}

Trigeminal Neuralgia Questionnaire. Protocol: Trigeminal Neuralgia, diagnostic/therapeutic mistakes (on behalf of Pain Panel European Academy of Neurology) 


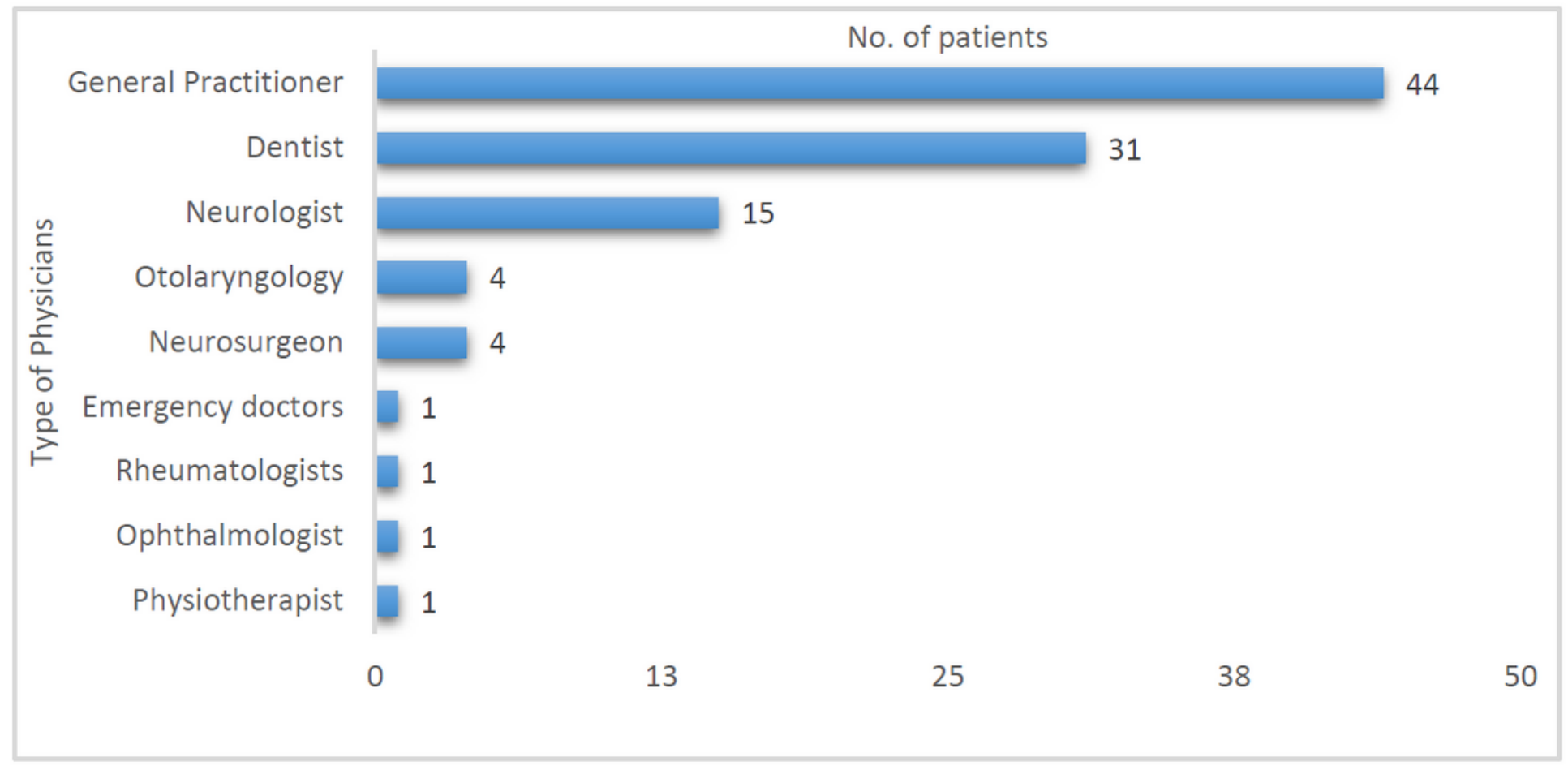

\section{Figure 2}

Physicians and specialists consulted by patients before being correctly diagnosed as suffering from classical trigeminal neuralgia.

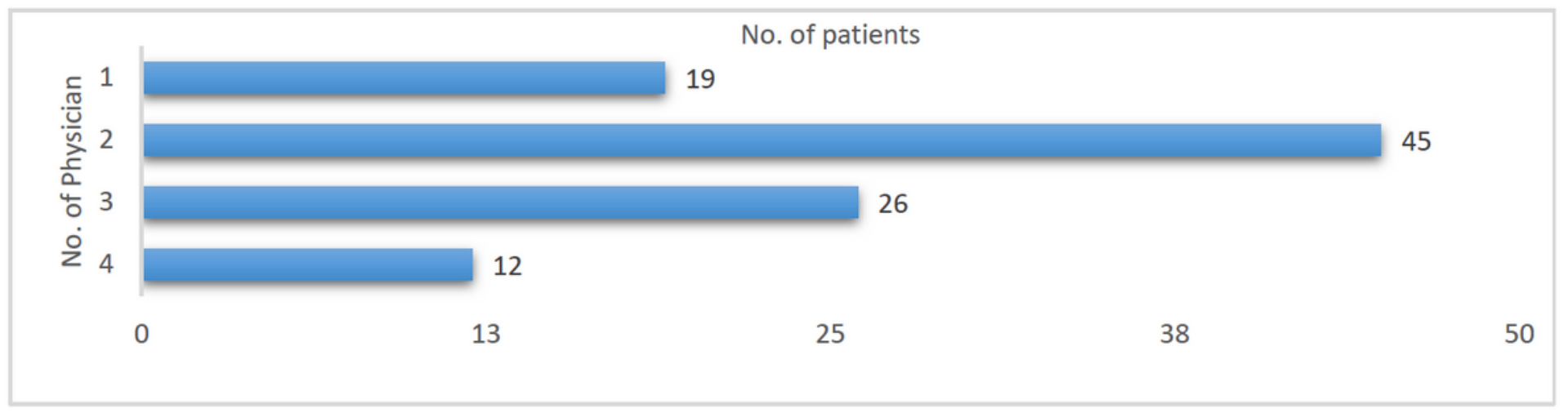

\section{Figure 3}

Number of physicians consulted by patients before being correctly diagnosed as suffering from classical trigeminal neuralgia. 


\begin{tabular}{|c|c|c|c|c|c|}
\hline \multirow{6}{*}{ 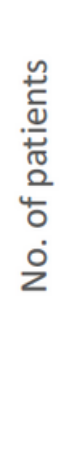 } & $\begin{array}{l}50 \\
40\end{array}$ & 35 & 39 & & \\
\hline & 30 & & & & \\
\hline & 20 & & & & \\
\hline & 10 & & & 10 & 5 \\
\hline & 0 & & & 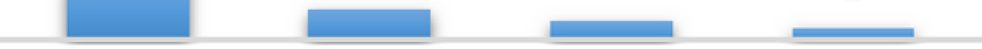 & \\
\hline & & $<1$ month & $2-6$ months & $\begin{array}{c}7 \text { - } 12 \text { months } 13 \text { - } 24 \text { months } 25 \text { - } 36 \text { months } 37 \text { - } 48 \text { months } \\
\text { Duration (months) }\end{array}$ & $>48$ months \\
\hline
\end{tabular}

\section{Figure 4}

Time elapsed between disease onset and first Headache Center consultation ("Patient Delay").

\begin{tabular}{|c|c|c|c|c|}
\hline \multirow{5}{*}{ 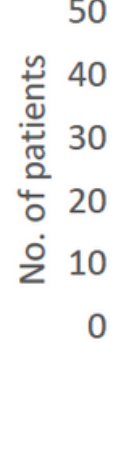 } & 36 & 40 & & \\
\hline & & & & \\
\hline & & & 14 & \\
\hline & & & 4 & 2 \\
\hline & $<1$ month & 2 - 6 months & $\begin{array}{c}7 \text { - } 12 \text { months } 13 \text { - } 24 \text { months } 25 \text { - } 36 \text { months } 37-48 \text { months } \\
\text { Duration (months) }\end{array}$ & $>48$ months \\
\hline
\end{tabular}

\section{Figure 5}

Time elapsed between disease onset and correct diagnosis ("Diagnostic Delay") 